\title{
Analysis of Charge Balance Control System for Lithium Ion Battery Pack
}

\author{
Zenghui Zhao, Wei Zhang, Ming Tang \\ School of Intelligence Science and Information Engineering \\ Xi'an Peihua University, Xi'an, China \\ 296883619@qq.com,25100423@qq.com,81408240@qq.com
}

Keywords: Lithium ion battery pack; New energy vehicles; Equilibrium control strategy; SOC estimate

\begin{abstract}
In recent years, lithium-ion battery packs are more and more widely used in new energy vehicles. In order to improve the battery life of new energy vehicles and to solve the imbalance of lithium-ion battery packs during charging, according to different operating conditions of lithium ion batteries, The voltage and SOC relationship between the proposed battery balancing control strategy, and lithium battery balancing control system hardware and software circuit design, and then set up a lithium-ion battery pack balance control circuit experimental model, and the experimental verification, test The results show that the proposed balance control strategy for lithium-ion batteries has a significant improvement on the inconsistency of a single cell, and the theoretical estimates are in good agreement with the authenticity.
\end{abstract}

\section{Preface}

With more and more attention paid to air pollution control in recent years, the new energy vehicle has developed rapidly and has become the best transportation to replace the traditional fuel vehicle, but the renewable capacity has been hindering the promotion of new energy vehicle. For the new energy vehicle, the electrical energy is mainly energy supplied by a large number of series lithium battery pack. In order to improve the renewable capacity of new energy vehicle, scholars have carried out a large number of theoretical and experimental studies on batteries, and found that one of the important factors affecting the lithium-ion battery is the inconsistency of the single cell in the lithium battery pack, that is, the problem of single cell will affect the efficiency of the whole battery pack, and then affect the efficiency of the lithium battery pack, and reduce the corresponding service life[1].

\section{Control Model of Lithium Ion Battery}

The inconsistency of single battery in the lithium battery pack, including the inconsistency of characteristics of the corresponding current and voltage, can seriously affect the efficiency of the entire battery pack, and then affect the utilization efficiency of lithium battery pack to reduce imbalance between lithium ion battery monomer battery for the corresponding service life of electric vehicle, the state can be monitored and identified, and balanced control can be carried out beyond the limit to reduce the matching imbalance between the cells. Thus, the efficiency and cycle life of lithium battery pack of new energy vehicle can be improved[2].

\subsection{Equilibrium Control Strategy}

The main goal of the control strategy of the lithium battery pack is to reduce the loss of the battery power, 
and the battery power loss is mainly energy dissipation and non-energy dissipation. The former consumes part of the energy in the form of heat, and the consumption rate is as high as 10 percent, and the disadvantage of energy loss is obvious. The latter can match the batteries with different energies, compensate the low energy batteries with high energy batteries, and reduce the overall energy loss. But the biggest problem with this approach is the complexity of circuit configuration design [3]. Therefore, based on the SOC algorithm of the battery pack, the cell consumption is estimated, and then the active balancing control strategy is used to control the battery pack energy.

\subsection{SOC Estimation of Lithium Ion Battery}

The estimation of lithium battery electric quantity is mainly carried out by SOC algorithm, and there are many factors affecting SOC, which are generally judged indirectly from internal and external factors [4]. The internal causes of SOC are caused by battery internal resistance and terminal voltage. External causes are caused by the internal current and temperature of the battery itself. The SOC algorithm of battery quantity is mainly defined as the ratio between the configurable power and the discharge capacity of the battery. The specific calculation equation is as follows:

$$
S O C=Q_{C} / C_{I}
$$

In the formula, $Q_{C}$ is the available energy of the battery pack, $C_{I}$ is the available energy during battery discharge.

In addition, the SOC algorithm of battery charge can also be defined as the ratio of battery charge to available power. The specific calculation equation is as follows:

$$
S O C=1-Q_{c} / C_{I}
$$

In the formula, $Q_{C}$ is full charge of the battery pack, $C_{I}$ is the overall available energy of the battery pack.

\subsection{The Balancing Control System of Lithium-ion Battery Series}

The balancing control system of lithium battery pack is mainly used to feedback the state information of lithium-ion battery with the on-board computer by the way of bus, so as to ensure the maximum energy and battery safe system of the battery [5]. The information module of lithium battery is formed by the control module, power module, gathering module, data communication module and SOC processing module. The balancing control system for the lithium battery pack mainly includes the following functions, (1) The real-time monitoring of the whole characteristics of battery pack estimates the intrinsic parameter of the battery pack to achieve the monitoring of the internal characteristics of the battery by analyzing the external characteristics of the battery, such as current, voltage, and so on, through a certain algorithm. The internal parameters of the battery pack are the basis of the balancing control, so it is very important to monitor the internal function from the outside. (2) On the basis of the first step to monitor the internal state of the battery pack, a series of management of the battery are carried out, such as heat, charge, discharge, balancing control, fault warning and so on. (3) the device of vehicle control system, charger, and display system are realized data exchange and sharing by establishing communication bus. 


\section{Hardware Circuit Design of Balancing Control System}

In this paper, the MC9S 12DG128, a 16-bit micro-controller of Free scale Company, is used as the control core of the lithium ion battery balancing control system, which is mainly including four parts of power module, gathering module, SOC processing module and digital function display module, of which the gathering module of temperature state is gathered by micro sensor, single-chip and data conversion module, the corresponding control strategy design is designed in accordance with the collected battery state information, of which the control module controls the state of the battery pack according to the acquisition signal and SOC processing module, so as to achieve the balanced charging of battery pack. The balancing module includes the MOSFET switch array, and the switch array is realized to switch the balancing module by the main controller. The selection of field-effect tube MOSFET shall follow various restrictive conditions and requirements. The balancing control system is selected, and the FDD8424 N \& P channel power MOSFET produced by Fair child Semiconductor company are adopted in this paper. The most notable feature of this model of the field-effect tube is that the MOSFET is integrated by a $\mathrm{N}$-channel and a P-channel MOSFET, and the integrated MOSFET parameters are as follows: the maximum current limit of $20 \mathrm{~A}$, the highest voltage of D/S pole is $40 \mathrm{~V}$. Beyond that, The MOSFET of the field-effect tube is made by a proprietary Power Trench process, this process has the advantage of low on-impedance of MOSFET, the conduction impedance is generally less than or equal to $30 \mathrm{~m} \Omega$, the conduction rise time is kept below $20 \mathrm{~ms}$, and the conduction drop time is also controlled below 20ms. The FET has the advantages of low on-impedance and fast turn-on time, which is very suitable for the application of balancing circuit. The balancing control circuit is shown in Figure. 1.

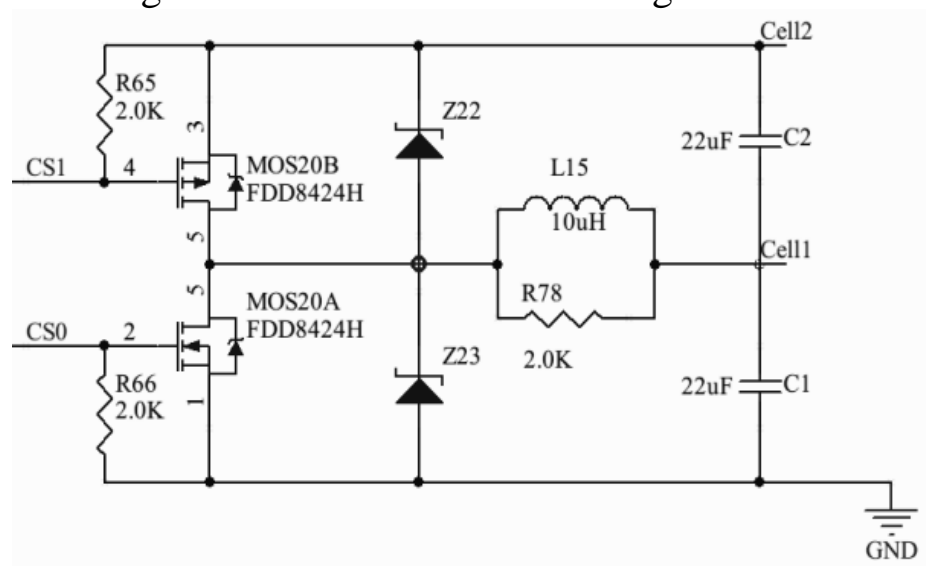

Figure 1 The Balancing Control Circuit

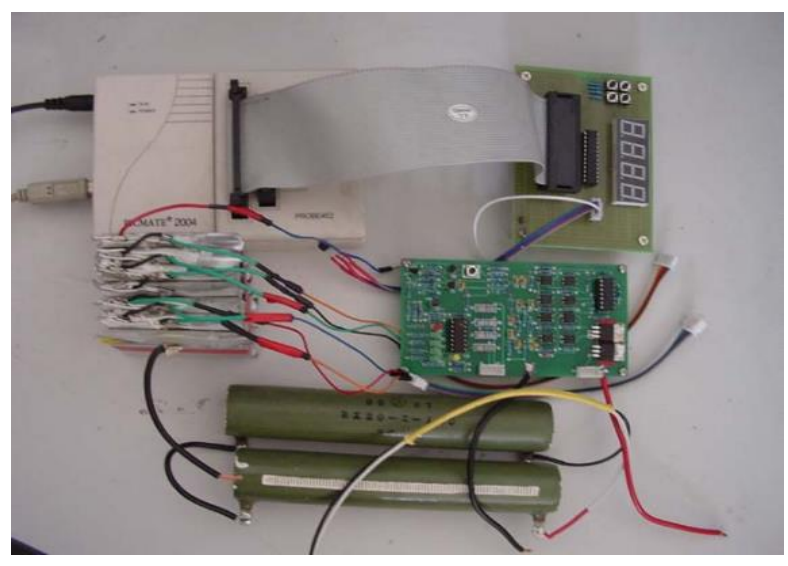

Figure. 2 The Balancing Control System experiment

\section{Experimental Results and Analysis}

According to the balancing control strategy and hardware and software circuit design, the corresponding test model is designed. The specific test model is shown in Figure. 2

\subsection{The Relationship Between Open-circuit Voltage and SOC}

According to the control circuit and corresponding SOC calculation method given in the aforementioned part of this paper, the data are collected through the test and the corresponding curve of voltage and SOC are plotted.

The detailed experimental conditions are as follows: the ambient temperature is $21 \sim 26{ }^{\circ} \mathrm{C}$ and 10 collection points are set. Firstly, the initial value is collected. In general, when the battery is discharged at a 
rate of $100 \mathrm{~mA}$ to a steady small current, the battery's power is completely released, and the initial value of the SOC battery can be solved by integral. The SOC values of 10 sampling points are collected. The battery is operated to discharge, and the discharge stops at a collection point, then the corresponding SOC1 value is calculated by the hour integration method, and then the discharge stops at the second collection points, and the corresponding SOC2 value is calculated by the hour integration method, by that analogy, the corresponding SOC10 value is calculated from the value of the tenth collection points. According to the voltage and SOC calculation value of the acquired multiple collection points, the corresponding curve is drawn, thus, the relationship between the voltage and working value of SOC is obtained.

\subsection{The Charging Curve}

The charging mode of lithium battery pack is composed of constant current charging and constant voltage charging. The charging current of the lithium battery is controlled between $400 \mathrm{~mA}-2 \mathrm{~A}$ and the minimum voltage is $3.35 \mathrm{~V}$, the temperature of the experimental environment is $23^{\circ} \mathrm{C}$, and the smallest capacity of the single cell is the initial capacity of the battery. Phase I: constant-current charging involves reducing the current to $600 \mathrm{~mA}$. When the charging voltage continues to increase and enter the constant voltage charging stage, thus the current is reduced to a low value and then stopped to charge. Based on the above experimental data and the obtained experimental results in section 3.1, the initial value of SOC0 of the battery can be calculated to be $8 \%$.

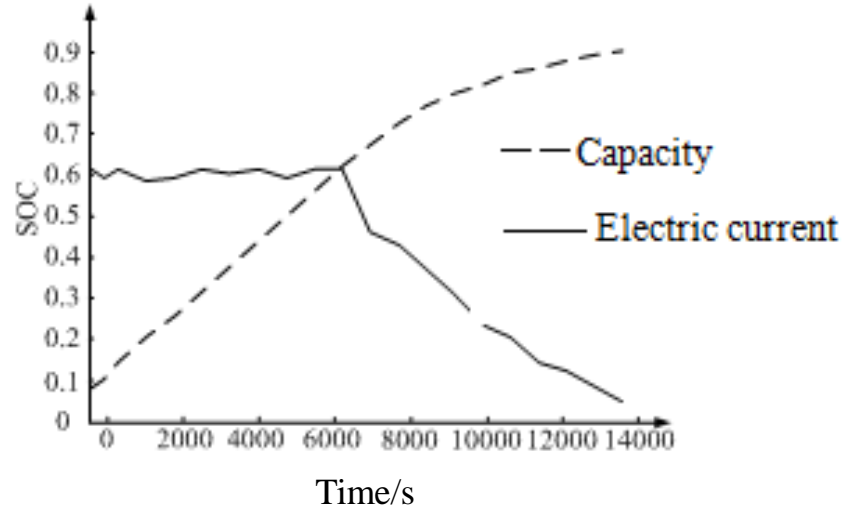

Figure. 3 Charging current and SOC curve

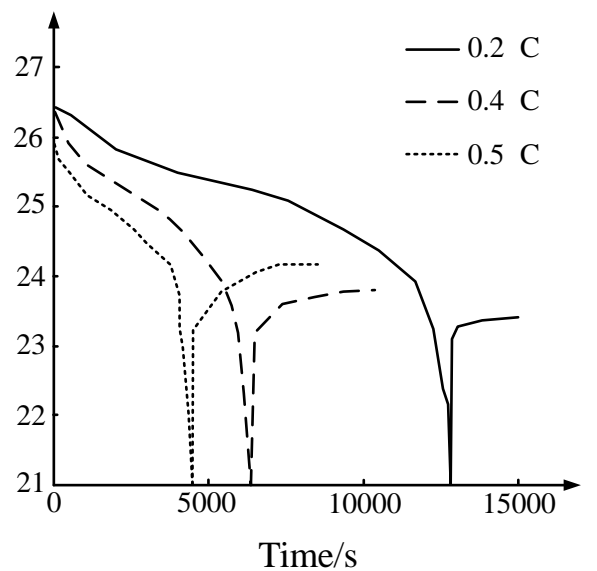

Figure.4 Voltage Curves at Different Discharge Rates

According to the experimental data, the charging current and SOC curve are shown in Figure.3. As you can see in Figure.3, the SOC value is a current of $0.3 \mathrm{C}$, and it takes about 4 hours to complete the battery charge. SOC value, voltage value increases with charging time at a certain slope in the first half of the constant current part, when the voltage value of the second half reaches the upper limit, the constant voltage charging voltage is carried, and the total voltage curve of SOC tends to moderate. In general, the initial stage of battery charging is the constant current stage, and the battery voltage rises rapidly. After a period of time, the voltage rise is linearized, and the rising amplitude tends to be stable until the second stage of constant voltage charging. The biggest advantage of the combination of constant current and constant voltage in this paper is that the battery charge saturation has the corresponding protection function, which can effectively prolong the battery service life.

\subsection{The Curve of Battery Constant-current Discharge}

The corresponding test environment for constant current discharge is $25^{\circ} \mathrm{C}$. The corresponding test conditions are as follows: the discharge rate is $0.2 \mathrm{C}$, current value is $400 \mathrm{~mA}$ and $0.4 \mathrm{C}$, current value is 
$800 \mathrm{~mA}$ and $0.5 \mathrm{C}$, current value reaches $1000 \mathrm{~mA}$ for constant current discharge. The voltage curve at different discharge rates are shown in Figure.4

As you can see from Figure.4, the discharge current has a continuous effect on the discharge state, and the specific results are as follows: the batteries with the same initial voltage are discharged with different constant current value. The time required to discharge from $0.2 \mathrm{C}$ to $21 \mathrm{~V}$ is about 3.6 hours, and from $0.4 \mathrm{C}$ to $21 \mathrm{~V}$ is about 2.2 hours. The time required to discharge from $0.5 \mathrm{C}$ to $21 \mathrm{~V}$ is about 1.3 hours. When the battery stop discharging is in the open-circuit state, because of the self-recovery effect of the battery, the terminal voltage of the battery rises gradually, and reaches the stable state after a certain time, in the meantime, as it can be seen from Figure.4, the larger the discharge current, the faster the voltage of the lithium battery decreases. Moreover, it can be seen from the figure that the discharge current is $0.5 \mathrm{C}>0.4 \mathrm{C}>0.2 \mathrm{C}$, the corresponding self-recovery voltage is $\mathrm{V} 0.5 \mathrm{C}>\mathrm{V} 0.4 \mathrm{C}>\mathrm{V} 0.2 \mathrm{C}$, in other words, the larger the discharge current, the higher the steady voltage of the corresponding lithium battery pack. According to the relationship between voltage and SOC, the magnitude of discharge current affects the stable voltage of lithium battery. The higher the voltage, the larger the battery capacity. Therefore, the cost curve between the residual charge and discharge current of the lithium battery can be obtained.

\section{Conclusion}

With the application of new energy vehicle more and more widely, as the main power supply of the new energy vehicle, the life of lithium electronic battery has become a hot topic. In order to solve the imbalance phenomenon in the charging process of Lithium-ion battery pack, according to the relationship between the voltage and SOC of Lithium-ion battery in different working conditions, the balancing control strategy of the battery pack is proposed in the paper, and the experimental model of the balancing control circuit of the lithium ion battery pack is designed and built, and the experimental results are verified. The experimental results show that the proposed balancing control strategy can improve the inconsistency of single battery obviously, and the theoretical estimation is in good agreement with the authenticity. The estimated value of the theory is in good agreement with the truthfulness.

\section{Reference}

[1] Stamps Andrew T, Holland Charles E, White Ralph E, et al. 2015, Analysis of Capacity Fade in a Lithium Ion Battery . Journal of Power Sourees, 150(15):229-239.

[2] Cai Xin, Li Bo. 2015, Research on SOC estimation of power battery based on neural network model, 36(01):12-18.

[3] Angel Kirchev.2015, Chapter 20-Battery Management and Battery Diagnostics Electrochemical Energy Storage for Renewable Sources and Grid Balancing, 411-435

[4] Humera Farooq. Muhammad Tariq Siddique. A Comparative Study on User Interfaces of Interactive Genetic Algorithm, Procedia Computer Science,32(2014)45-52.

[5] Rutuparna Panda, Manoj Kumar Naik. A novel adaptive crossover bacterial foraging optimization algorithm for linear discriminant analysis based face recognition, Applied Soft Computing, 30(2015)722-736. 